\title{
LA PARTICIPATION DANS UN MONDE DE COMMUNICATION
}

\author{
INTRODUCTION
}

Sandrine Roginsky ${ }^{1}$, Damien RenarD ${ }^{2}$, Marie DufraSNE ${ }^{3}$

«Participez aux consultations citoyennes", «Participez à la levée de fonds », Participez à des concours d'idées », «2015 façons de participer », « Participez ! »... Les injonctions à la participation sont devenues comminatoires tels des impératifs catégoriques, quel que soit le domaine d'activité. Les problématiques de participation submergent ainsi les discours et les pratiques, et ne sont pas sans interroger les visions de la communication qu'elles mobilisent. En cela, elles font écho aux réflexions développées par Dewey qui voit dans la participation un critère pour hiérarchiser le degré de communication (Quéré, 2014). Plus le degré est élevé et la forme de communication élaborée, plus sa portée sociale serait significative. Participation et communica-

1 Sandrine Roginsky est professeure à l'École de communication de l'Université catholique de Louvain et directrice du LASCO.

2 Damien RenARD est professeur à l'École de communication de l'Université catholique de Louvain.

3 Marie Dufrasne est professeure en Information et Communication à l'Université Saint-Louis-Bruxelles.

Recherches en communication, $\mathrm{n}^{\circ} 52$ - Article publié le 08/09/2021 
tion renvoient ainsi à l'idée d'un lien universel, à l'idéal de « fusion communautaire » (Mattelart, 1999) ou de « communication planétaire » (Breton, 1992). Les imaginaires de la communication comme lien universel ou moyen du vivre-ensemble se mêlent à ceux de la participation comme idéal d'échange, de partage, de débat et de concertation.

Pour saisir ce glissement, ce numéro propose de replacer la participation - active, multiple et ambivalente - au cœur de ce processus de médiatisation croissante des relations sociales. C'est ainsi à la lumière des sciences de l'information et de la communication qu'il s'agit ici de (re)visiter et (ré)interroger le concept de participation, sans néanmoins mettre de côté sa circulation dans différentes disciplines de recherche. Dans le cadre de ce dossier, nous envisageons en effet la participation en tant que « concept-frontière », par transposition de la notion d'objetfrontière (Star, 2010), c'est-à-dire en tant que concept rhizomatique à l'intérieur duquel différents points de vue se rencontrent et coopèrent. Au-delà des différences disciplinaires, explorer la participation avec une approche communicationnelle amène à interroger des concepts périphériques comme la mobilisation ou encore l'engagement.

À travers ce dossier, il s'agit finalement d'interroger la tension sous-jacente entre processus de participation et instrumentalisation rhétorique et/ou technique de la participation mais également de discuter des implications que cet objet dynamique et flexible peut engendrer. Cette tension traverse en effet l'ensemble des contributions qui constituent ce dossier. La participation y apparaît ainsi organisée, « calculée », orchestrée, parfois mise en scène, sans pour autant s'y réduire : sa portée sociale ne se réduit en effet pas uniquement à la création d'un simulacre. En se présentant comme une forme d'idéal à atteindre, la participation donne ainsi à voir la place centrale conférée à la dimension relationnelle des activités sociales.

Le premier article du numéro nous amène à la télévision, dans le cadre de laquelle Céline Ségur questionne les manifestations et les enjeux d'un idéal participatif. Dans ce contexte, le participatif est présenté comme un modèle dans lequel les producteurs et productrices et les consommateurs et consommatrices de médias sont en interaction les un·es avec les autres. Cet idéal n'est pas nouveau, propre à la période de digitalisation (Couldry \& Hepp, 2016) dans laquelle nous nous trouvons. Au contraire, l'auteure constate que cet idéal participatif a émergé dès les années 1950 et, depuis, a pris différentes formes. 
L'auteure montre d'ailleurs comment les formes participatives sont au service d'ambivalences qui caractérisent les objectifs des diffuseurs entre notamment un travail relationnel auprès des publics et un travail de fidélisation de type commercial. Cette situation amène finalement à survaloriser la participation dans les dispositifs télévisuels.

*

Une fois posé cet idéal en toile de fond, les autres articles du dossier proposent différents éclairages et déclinaisons de la participation.

En contexte organisationnel, en premier lieu, puisqu'il s'agit dans le second article du dossier d'interroger la question de la participation à partir d'une recherche qualitative au sein de la section française d'une ONG internationale, Amnesty International France (AIF). Dans ce contexte, comme le montre Vincent Brulois, la participation est envisagée comme une modalité de coordination d'acteurs sociaux au statut diversifié, puisque certain 'es sont bénévoles, d'autres militant·es et d'autres salarié·es. Et si c'est a priori d'abord la question de l'engagement qui semble se poser pour une organisation de ce type, l'auteur montre que c'est finalement moins la question de l'engagement que celle de la participation à laquelle est confrontée AIF - notamment la participation des bénévoles. Or, celle-ci induit la mise en place d'une nouvelle structure dans l'organisation; ce qui a pour conséquence une remise en cause des équilibres internes. La participation des bénévoles ne se fait pas sans tensions, comme peuvent par exemple le donner à voir les biens nommés « ateliers de la controverse » dans lesquels il est possible de constater que derrière la question de la participation, c'est aussi (et d'abord ?) une recherche de reconnaissance qui transparait. Ce sont certes les difficultés à faire vivre la participation qu'il est possible de mieux appréhender à la lecture de cet article, mais aussi l'importance du cadre qui tout à la fois permet et favorise la participation mais la contraint également.

En effet, il ne semble pas pouvoir y avoir participation sans dispositif organisant un tant soit peu les règles de l'échange, comme l'illustre l'article de Christel Christophe. Celui-ci décrit la manière dont l'équipe d'un centre d'accompagnement à l'entrepreneuriat social organise et met en œuvre la participation au moyen d'« arrangements spatiaux et sémiotiques » (Meunier, 1999) qui définissent des espaces de participation spécifiques et circonscrits. Les membres de l'équipe jugent la compétence d'animation de groupe comme stratégique et estiment donc utile de s'y former. Les dispositifs participatifs ainsi conçus 
créent des " traces d'expériences collectives 》 et sont pourvoyeurs de données qui contribuent à la connaissance de l'organisation et ses publics, et ce faisant à l'apprentissage organisationnel.

Après l'exploration de la participation en contexte organisationnel, c'est à la participation telle qu'elle s'envisage et se déploie sur internet que les articles suivant s'intéressent.

Ainsi, l'article de Christine Chevret-Castellani montre comment les formes de mobilisation dans le contexte de manifestations contre un article spécifique d'une directive européenne sur le droit d'auteur peuvent être influencées par les plateformes sur lesquelles elles se déploient. En l'occurrence, ici, l'auteure s'intéresse aux usages de la plateforme YouTube, utilisée pour donner à voir les manifestations en question; ce qui l'amène à interroger les évolutions des significations de la participation en partant du postulat que celle-ci n'est pas réductible à la contribution citoyenne réalisée sur des dispositifs mis en place par des pouvoirs publics. Elle montre que les vidéos témoignent d'une forme de démocratisation par la revendication de la compréhension d'un texte juridique technique. Ce faisant, il est possible de voir une construction d'un commun, voire d'un collectif, émerger, mais dont le formatage est néanmoins induit par la plateforme qui appartient à une entreprise américaine, elle-même directement concernée par la directive européenne contestée et mobilisée également pour défendre ses propres intérêts économiques; intérêts économiques certainement peu en phase avec les intérêts défendus par les manifestants qui utilisent néanmoins son espace pour donner de la visibilité à leurs actions.

C'est aussi à l'espace de participation politique d'une plateforme qui n'a pas été imaginée pour cela que Édouard Bouté et Clément Mabi s'intéressent. Ils posent leur regard sur une plateforme numérique de « social coding », GitHub, qui permet la collaboration de féru·es de l'informatique, et tentent de voir si l'on y trouve des « tiers espaces », autrement dit des espaces qui a priori ne sont pas destinés à accueillir des formes de participation politique mais qui peuvent néanmoins les favoriser. Ils montrent ainsi que si la plateforme peut certes être le support d'une participation politique, celle-ci est fragile et très cadrée par ce qu'ils appellent une " police normative » exercée par certains membres de la plateforme, en fonction notamment de leur place dans la hiérarchie et du pouvoir que celle-ci leur confère. 
Ce sont donc des visions contrastées de la participation que ces différents exemples empiriques donnent à voir.

L'article de Caroline Marti à cet égard offre un autre cas d'étude tout aussi riche et intéressant, puisqu'il s'agit ici de s'intéresser à des plateformes numériques qui proposent le référencement de restaurants à partir de la collecte des avis de consommateurs et consommatrices. L'auteure montre en premier lieu comment ces avis - ici envisagés comme expressions individuelles qui deviennent contributions par le truchement de leur publicisation sur ces plateformes - s'ils sont a priori hétérogènes et spontanés, se trouvent néanmoins cadrés par le système d'écriture sur lequel ils se déploient. Loin d'être la seule tension observée dans son exploration, l'auteure note aussi comment l'avis se trouve être tout à la fois «l'expression de soi au service d'une communauté et au service de la consommation ", mais également au service des plateformes. Ce faisant, c'est le lien entre avis et participation qui devient apparent, le premier permettant de donner à voir la seconde - et plus les avis sont nombreux, plus la participation serait intense dans la grammaire des plateformes, qui en cela reprend des arguments quantitativistes qui la dépassent (voir par exemple Desrosières, 2008). L'auteure de parler ainsi d'un « capitalisme participatif », qui voit dominer une visée utilitaire et instrumentale de la participation.

Cette observation permet certainement d'éclairer l'étude empirique proposée par Lucie Delias dans l'article qui suit. Celle-ci s'intéresse à des sites français d'organisation de sorties et de rencontres amoureuses qui s'adressent à des publics d'âge divers, en mettant dans le cadre de sa recherche la focale sur les publics plus âgés. Elle montre ainsi que les discours et les fonctionnalités des sites de rencontres construisent un public imaginé à partir de représentations du «bon vieillissement » qui finalement finissent par proposer une conception de la participation qui ne correspond que partiellement aux pratiques des internautes âgés. Le " bon usage » des outils numériques y est présenté à la fois comme un levier et un indice du « bien-vieillir ». Autrement dit, les usagers âgés d'internet sont désormais sujets aux injections à participer en ligne, mais au moyen de dispositifs fort contraignants qui ne leur correspondent pas nécessairement.

L'article proposé par Victor Wiard, qui conclut le dossier, fait un pas de côté et propose une approche un peu différente, puisqu'il s'agit ici de proposer une grille d'analyse des pratiques des acteurs impliqués dans la production de nouvelles locales sur internet. Ainsi cette grille, qui se présente comme un « modèle ADD élargi » (ADD pour accès, 
dialogue et délibération), propose de décentrer l'analyse de la participation aux médias en se concentrant non pas sur une vision stricte du «journalisme participatif» mais en intégrant au contraire la multiplicité des acteurs sociaux et des manières dont ceux-ci prennent part à la production de contenus liés à l'actualité.

$*$

Les articles du dossier montrent tous, quel que soit le contexte et la nature de la participation, que celle-ci est le produit d'interactions sociales qui se déploient dans des espaces configurés par des agencements d'éléments - qu'il s'agisse de discours, de procédures ou d'équipements techniques. Sans acteurs sociaux, pas de participation - ce que montrent d'ailleurs bien les articles qui questionnent l'agentivité des " participant·es » au sein des cadrages socio-techniques - mais sans cadrage des activités de ces acteurs sociaux pas de participation non plus. Ce sont finalement ces deux dimensions que les articles explorent, en les articulant à chaque fois - ce qui permet ainsi de dépasser des approches techno-déterministes ou fonctionnalistes. Il est ainsi possible de repérer la façon dont la participation devient un idéal inscrit dans la configuration tout à la fois des agencements et des interactions que ceux-ci encadrent.

Quel que soit le contexte et/ou le secteur professionnel concerné, la participation se présente tout à la fois comme un objectif en tant que tel à atteindre mais également comme un moyen pour arriver à une fin - qui dépasse donc ici la seule participation. Il peut s'agir de permettre l'engagement, la mobilisation et/ou l'inclusion, de conférer de la légitimité à des acteurs et/ou un dispositif particulier, de favoriser la réussite économique, etc. En définitive, explorer les modalités et les discours sur la participation nous renseigne non pas tant sur la participation en tant que telle, ou du moins pas seulement, mais aussi et surtout sur les contextes dans lesquels elle se construit, les acteurs sociaux impliqués (soit en tant que concepteurs et conceptrices, soit en tant qu'utilisateurs et utilisatrices) et les manières dont ceux-ci prennent part à son cadrage et à sa configuration.

\section{Références}

Breton, P. (1992). L'Utopie de la communication. Le mythe du village planétaire. Paris : La Découverte.

Couldry, N. \& Hepp, A. (2016). The Mediated Construction of Reality. Cambridge: Polity Press. 
Desrosières, A. (2008). Pour une sociologie historique de la quantification. L'argument statistique. Paris : Presses des Mines.

Mattelart, A. (1999). Histoire de l'utopie planétaire : de la cité prophétique à la société globale. Paris : La Découverte.

Meunier, J. P. (1999). Dispositif et théories de la communication : deux concepts en rapport de codétermination. Hermès, 25(3), 83-91. Doi : https://doi. org/10.4267/2042/14976

Quéré, L. (2014). Le modèle esthétique de la communication de John Dewey. Colloque «Communication, culture et communauté. L'École de Chicago en débat ». Universidade Lusofona do Porto (Portugal), 21 février 2014. Disponible à : http://cems. ehess.fr/docannexe/file/3168/porto2014_quere2.pdf

Star, S.L. (2010). This is Not a Boundary Object: Reflections on the Origin of a Concept. Science, Technology \& Human Values, 35(5), 601-617. Doi : https://doi. org/10.1177/0162243910377624 


\section{(c) (1) (8) $\Theta$}

«Attibution - pas d'utilisation Commerciale - Pas de Modification 4.0. International» (CC BY-NC-ND) 